\title{
Photodegradation of photodynamic therapy agents in aqueous $\mathrm{TiO}_{2}$ suspensions
}

\author{
A. S. Oliveira ${ }^{1,2}$, C. G. Maia ${ }^{1}$, P. Brito ${ }^{1}$, R. Boscencu ${ }^{3}$, \\ R. Socoteanu ${ }^{4}$, M. Ilie $^{3} \&$ L. F. V. Ferreira ${ }^{2}$ \\ ${ }^{1}$ Centro Interdisciplinar de Investigação e Inovação, C3I, \\ Escola Superior de Tecnologia e Gestão, \\ Instituto Politécnico de Portalegre, Portugal \\ ${ }^{2}$ Centro de Química-Física Molecular e Instituto de Nanociencias e \\ Nanotecnologia, Instituto Superior Técnico, \\ Universidade Técnica de Lisboa, Portugal \\ ${ }^{3}$ Faculty of Pharmacy, "Carol Davila" University of Medicine \\ and Pharmacy, Romania \\ 4"Ilie Murgulescu” Institute, Romanian Academy, Romania
}

\begin{abstract}
The presence of emergent pollutants in waters and wastewaters are an issue of increasing concern due to the risk they pose to human and environmental health. Cancer Photodynamic Therapy photosensitizers and their metabolites and photodegradation products are pharmaceutical substances that after treatment will be eliminated from the human body and will eventually reach water bodies. Porphyrins are well established PDT sensitizers and cyanine dyes are promising candidates for the same use. In that way it is important to know how those compounds degrade once they reach water bodies and to find efficient treatment methodologies in case they are persistent. In this paper the simulated solar light photodegradation of a porphyrin (Zinctetraphenylporphyrin) and of a cyanine dye (3,3'-diethylindocarbocyanine iodide) in water was investigated, in the absence and in the presence of the most used photocatalyst for semiconductor photocatalysis: Titanium dioxide $\left(\mathrm{TiO}_{2}\right)$. We observed that the porphyrin $\left(1 \times 10^{-3} \mathrm{M}\right)$ did not undergo photodegradation in the absence of photocatalyst and that in its presence the photodegradation process was strongly promoted $(60 \%$ photodegradation reached after 1 hour of irradiation with $0,01 \mathrm{~g}$ of $\mathrm{TiO}_{2}$ ). The
\end{abstract}


cyanine dye $\left(1 \times 10^{-3} \mathrm{M}\right)$ suffered a photodegradation of about $20 \%$ even in the absence of $\mathrm{TiO}_{2}$ and reached $100 \%$ photodegradation in its presence.

Keywords: emergent pollutants, wastewater treatments, Porphyrins, cyanine dyes, photodynamic therapy sensitizers (PDT), advanced oxidation processes (AOPs), heterogeneous photocatalysis, $\mathrm{TiO}_{2}$, solar photocatalysis.

\section{Introduction}

Nowadays wastewater may contain several different pharmaceuticals including antibiotics, hormones and other endocrine disruptors, chemotherapy and photodynamic therapy medicines, antipyretics, etc. Those are present in municipal sewage, largely as a result of human use and/or excretion. Much of the concern regarding the presence of these substances in wastewater and their persistence to wastewater treatment processes is because they may contribute to directly or indirectly affect the environmental and human health [1, 2]. For most of these substances their potential highly harmful effect on environmental and human health is just starting to be perceived, and the correct extension of their impacts is still far from being fully understood; reason why these substances are generically referred as emerging pollutants. Conventional water and wastewater treatment are inefficient for substantially removing many of them [1-3].

The photodegradation and mineralization of several emerging pollutants, namely different pharmaceuticals is currently been widely studied because of the danger their present or that of their residues in water bodies represent to the environment and also due to their highly recalcitrant character. Research has show that advanced oxidative processes, which generate very active oxidative species such as the hydroxyl radicals, are promising tools for the destruction of pharmaceuticals compounds [3-5]. While there appears to be no standard treatment for removal of all residual pharmaceuticals under conventional treatment processes, there is a strong opinion that advanced oxidation processes can be used for their effective removal [3-5].

The advanced oxidative processes (AOP's) are an alternative treatment for wastewater containing persistent and biorecalcitrant pollutants [3 and references quoted there]. Fujitsu and Honda [6] discovery of the photoinduced water cleavage by titanium dioxide $\left(\mathrm{TiO}_{2}\right)$ heterogeneous photocatalytic oxidation using the semiconductor as catalytic. The semiconductor $\mathrm{TiO}_{2}$ presented advantageous such as simplicity, low cost, high photochemical reactive, stability in aqueous systems and low environmental toxicity, while promoting efficiently the degradation of very persistent target organic compounds and industrial effluents $[7,8]$.

In recent few years the use of Porphyrins in biomedical applications increased exponentially. Porphyrins are structures that mimic naturally occurring compounds and promising candidates as fluorescent markers. This new field of use for porphyrins was partially steamed by their application as sensitizers in photodynamic therapy (PDT), exhibiting convenient absorption in the phototherapeutic window ( $\sim 70$ to $1100 \mathrm{~nm}$ ). Focus on all classes of NIR probes was mostly triggered by the development of reliable and inexpensive NIR 
emitting laser diodes. The gallium-arsenic semiconductor laser (780-830nm) made these probes highly efficient absorbers, mainly for medical applications, as fluorescent NIR probes avoid interference with background fluorescence of biomolecules. NIR probes are also suitable for biological applications, since light of longer wavelengths penetrates the tissue more easily and without interferences, making them more attractive for in vivo measurements [9-12]. Although less used in PDT than porphyrins, several cyanine dyes emitting in the same region have been also recently reported as promising candidates for use as PDT sensitizers [13-15].

The aim of this work is to know how near infrared porphyrin and cyanine dyes fade after being discharged in wastewaters following their biomedical uses and investigate if advanced oxidative processes can be useful on promoting and accelerate their photodegradation on wastewaters. Titanium dioxide $\left(\mathrm{TiO}_{2}\right)$ heterogeneous photocatalysis was the AOP treatment selected. Semiconductor photocatalysis by is an alternative method for conventional water and wastewater treatment technologies being able to mineralise to $\mathrm{CO}_{2}$ and $\mathrm{H}_{2} \mathrm{O}$ the most recalcitrant compounds. This methodology has the advantage that it can be sensitized by solar light what represents a major energy cost saving when developing a water and wastewater remediation treatment. $\mathrm{TiO}_{2}$ is a photostable, inexpensive and non toxic material. The most popular titanium dioxide used is P25 produced by Degussa Company [3].

\section{Experimental section}

\subsection{Reagents and materials}

Zinctetraphenylporphyrin (ZnTPP) and 3,3'-diethylindocarbocyanine iodide were purchased from Aldrich in the highest purity available.

The photocatalyst used was titanium dioxide, Degussa P25 (80\% anatase), is the most commonly used due to its high photoactivity when compared to other sources. This is due to its high surface area about $50 \mathrm{~m}^{2}$ to its crystalline complex microstructure and promotes better separation of inhibiting the loads recombination. All the other chemicals used in this study were obtained from Aldrich. We used bidistilled water prepared in the laboratory.

\subsection{Experimental procedure}

Fresh solutions were always prepared before used and diluted according to the requirements of the experiments. Photodegradations were performed with $50 \mathrm{ml}$ of solutions prepared with the porphyrin and the cyanine in distilled water. Before irradiation each sample was placed in a static mixer, in the dark for 40 minutes. Then the solutions were placed under irradiation, in static mixer, to keep the solution homogeneous.

For the study of the influence of $\mathrm{TiO}_{2}$ photocatalyst concentration of the photodegradation efficiency $50 \mathrm{ml}$ of a $0,01 \mathrm{gL}^{-1}$ dye solution of each of the studied dyes containing different concentrations of $\mathrm{TiO}_{2}\left(0,1 \times 10^{-3}\right.$ and 
$1 \times 10^{-2} \mathrm{gL}^{-1}$ ) were irradiated in the photoreactor described below for $300 \mathrm{~min}$. Samples were collected at the times, 0, 15, 30, 45, 60, 90,120, 180 and 300 minutes for analysis in UV-Visible.

The photodegradation of the samples of the porphyrins and cyanine dyes were evaluated spectrophotometrically measuring the maximum absorption at the corresponding wavelength of each dye (respectively $\lambda_{\max }=422 \mathrm{~nm}$ for ZnTPP and $\lambda_{\max }=477 \mathrm{~nm}$ for 3,3'-diethylindocarbocyanine iodide). A double beam UVVisible spectrophotometer (Cary 100 Bio) was used for spectrophotometric determinations of the absorption spectra of the dyes from 200-800 nm. Spectra of the dyes in water were recorded with $1 \mathrm{~cm}$ or $1 \mathrm{~mm}$ quartz cuvettes.

The samples were irradiated with a 125 Watts mercury vapour lamp (from Osram). In this type of commercial lamps, the filament is protected by a glass bulb that cuts all UV-A and UV-B radiation. The glass bulb presents a white colour due to the internal phosphor coating that improves the radiation of the lamp on the visible region. This type of lamps with glass bulb is appropriated to selectively illuminate and exclusively excite $\mathrm{TiO}_{2}$ band gap, avoiding direct photolysis of the dye molecules that could be simultaneously promoted if all the lamp emission profile was available.

The photoreactor were the samples undergo irradiation is composed by an elliptical cover that supports the irradiation source described above and of a base containing a magnetic stirrer, where the samples to be irradiated are placed in $100 \mathrm{~mL}$ beakers. The light arising from the mercury lamp was measured at $366 \mathrm{~nm}$ (the wavelength of $\mathrm{TiO}_{2}$ bandgap [3]) with the help of a Cole Parmer radiometer (series 9811-50) placed above the beaker with the sample to be irradiated. All samples were illuminated with an average irradiation power of $2.7 \mathrm{~mW} / \mathrm{cm}^{2}$.

\section{Results and discussion}

\subsection{Effect of $\mathrm{TiO}_{2}$ photocatalyst concentration on Porphyrin photodegradation}

Figure 1 displays the results of the photodegradation of ZnTPP in water. Figure 1a) present the results of the porphyrin irradiation in the absence of $\mathrm{TiO}_{2}$ while Figure $1 \mathrm{~b}$ ) presents the results of the porphyrin irradiated with $0,01 \mathrm{~g}$ of $\mathrm{TiO}_{2}$.

In Figure 1a) we can observe that ZnTPP in water doesn't experiment any significant photodegradation during the 5hours irradiation period in the absence of the photocatalyst while in part b) of the same figure we see the porphyrin undergo a significant photodegradation in the same period. For a smaller amount of $\mathrm{TiO}_{2}$ added the photodegradation observed was much smaller.

Figure 2 displays the percentage photodegradation of the compound calculated as

$$
\text { Photodegradation }=\left(\frac{A b s i-A b s f}{A b s i}\right) * 100 \text {. }
$$



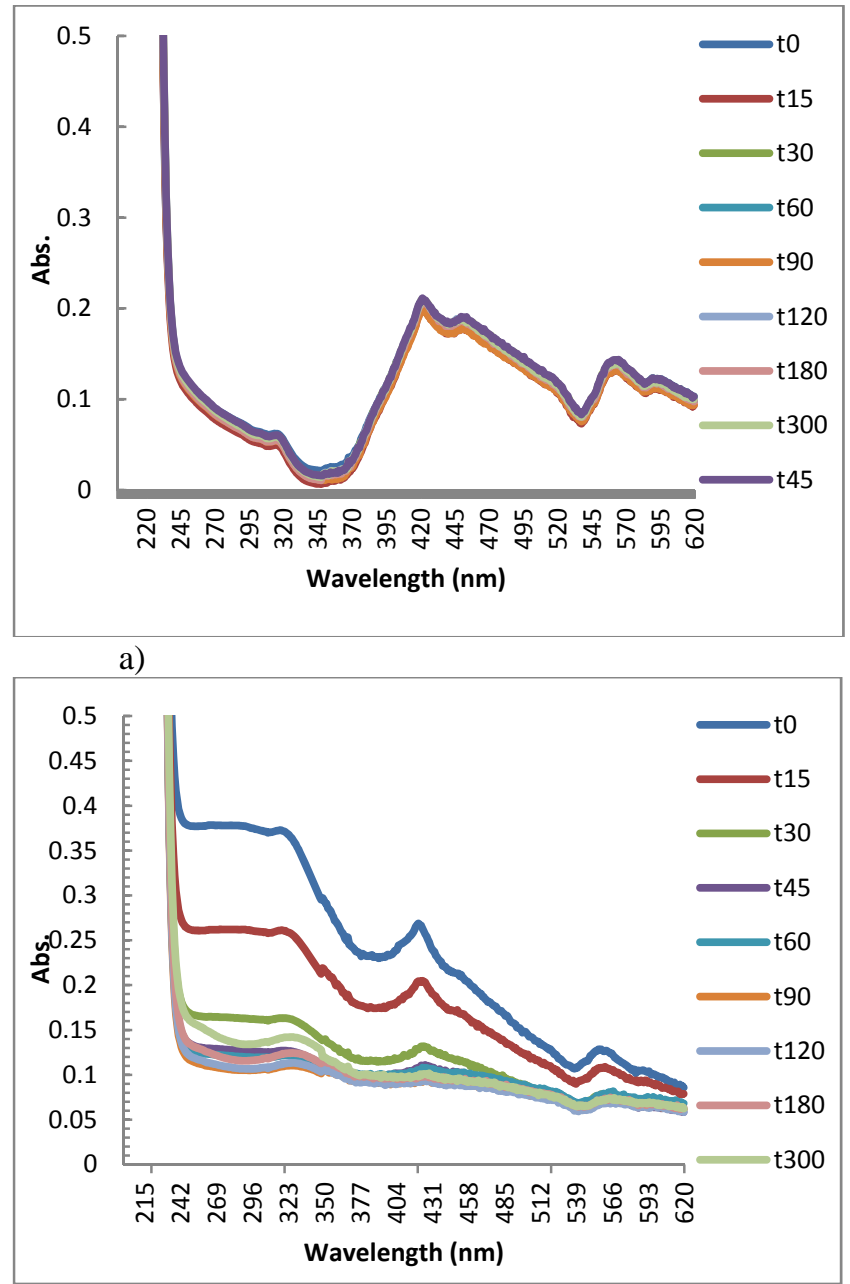

b)

Figure 1: Ground state absorption spectra for Zinctetraphenylporphyrin in water $\left(1 \times 10^{-3} \mathrm{M}\right)$ under a 125 watt mercury vapour lamp irradiation over time. Spectra were register after 0, 14, 30, 45, 60, 90, 120, 180 and 300 minutes of irradiation in a) without and b) with $\mathrm{TiO}_{2}$.

Contrarily to the commonly observed to several dyes [8] for this porphyrin in water the total photodegradation of the dye wasn't reached in the total irradiation time. This is probably due to the fact that porphyrins are mostly insoluble in water. Even though it was possible to conclude that the efficiency of photocatalytic degradation of ZnTPP in water was clearly increased by the addition of photocatalyst and by increasing its amount. 


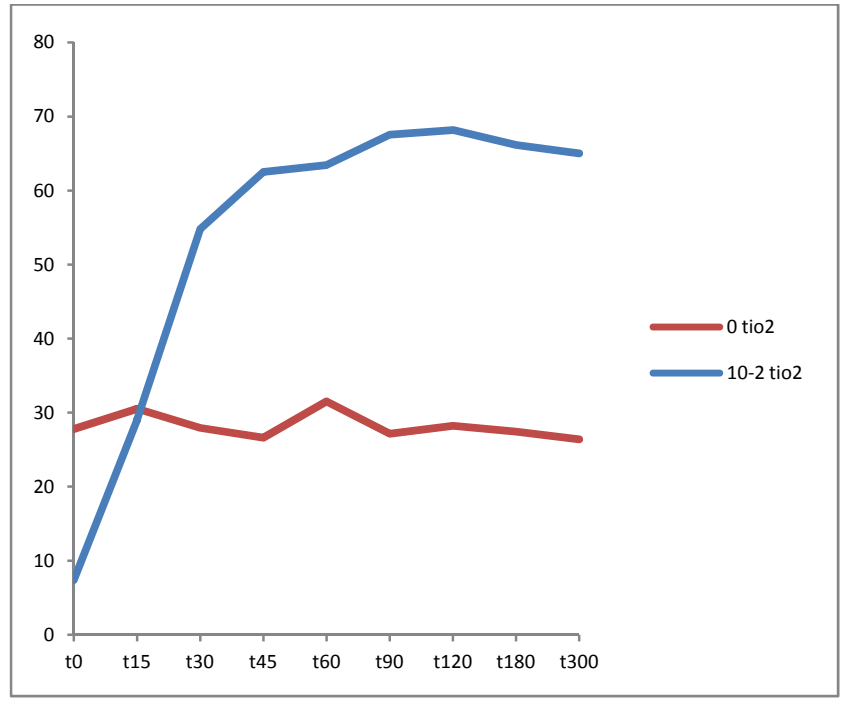

Figure 2: Percentage photodegradation obtained for Zinctetraphenylporphyrin in water $\left(1 \times 10^{-3} \mathrm{M}\right)$ under a 125 watts mercury vapour lamp irradiation over time (respectively 0, 14, 30, 45, 60, 90, 120, 180 and 300 minutes of irradiation without and with $\mathrm{TiO}_{2}$.

\subsection{Effect of $\mathrm{TiO}_{2}$ photocatalyst concentration on cyanine photodegradation}

Figure 3 displays the results of the photodegradation of 3,3'-diethylindocarbocyanine iodide in water. Figure 3a) presents the results of the cyanine irradiation in the absence of $\mathrm{TiO}_{2}$ while Figure 3b) presents the results of the cyanine irradiated with $0,01 \mathrm{~g}$ of $\mathrm{TiO}_{2}$.

As can be seen from Figure 3a) this cyanine dye in water experiments some photodegradation even in the absence of titanium dioxide. The dye slowly loses its colour during the time of the experiment as shows the neat decrease of the maximum of the absorption spectrum of the cyanine in water after 5 hours of irradiation. When $1 \times 10^{-2} \mathrm{gL}^{-1}$ of $\mathrm{TiO}_{2}$ were added to the solution to be remediated, a much consistent and quick lost of colour can be observed. By the end of 300 minutes of irradiation, the typical visible absorption band of the cyanine dye disappeared completely. After treatment with the photocatalysts only their absorption bands in the UV region on the absorption spectra are still present.

Figure 4 displays the percentage photodegradation of the cyanine dye in water in the presence and in the absence of the photocatalyst.

From Figure 4 it can be observed that cyanine dye alone degraded around $20 \%$ while in the presence of $0,01 \mathrm{~g}$ of $\mathrm{TiO}_{2}$ the degradation reached $100 \%$ in the 300 minutes of degradation. In the remaining smaller concentrations of $\mathrm{TiO}_{2}$ studied, it was found that the degradation was less efficient. 


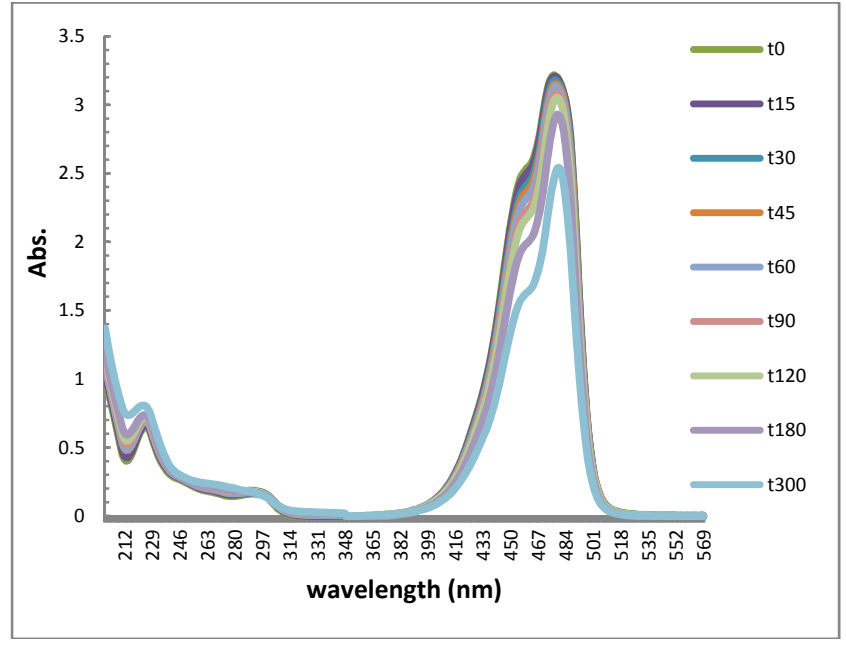

a)

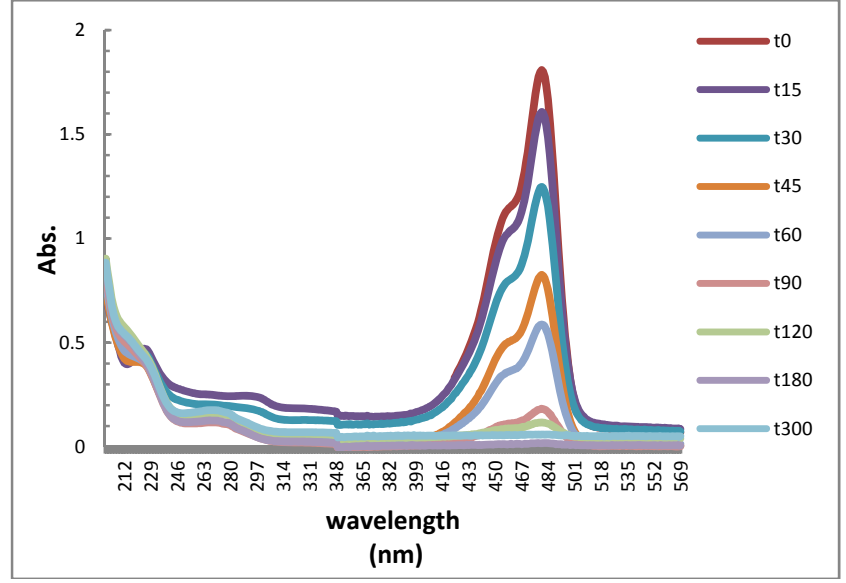

b)

Figure 3: Ground state absorption spectra for 3,3-diethylindocarbocyanine iodide in water $\left(1 \times 10^{-3} \mathrm{M}\right)$ under 125 watt mercury vapour lamp irradiation over time. Spectra were register after $0,14,30,45,60$, 90, 120, 180 and 300 minutes of irradiation in a) without and b) with $\mathrm{TiO}_{2}$. 


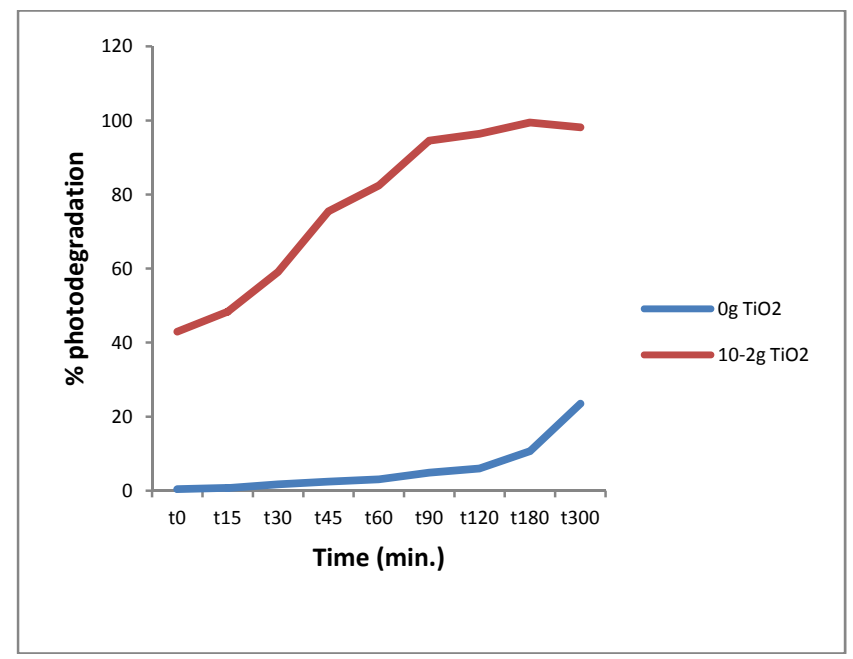

Figure 4: Percentage photodegradation obtained for 3,3'diethylindocarbocyanine iodide in water $\left(1 \times 10^{-3} \mathrm{M}\right)$ under a 125 watts mercury vapour lamp irradiation over time (respectively 0 , 14, 30, 45, 60, 90, 120, 180 and 300 minutes of irradiation without and with $\mathrm{TiO}_{2}$.

\section{Conclusions}

In this study it was concluded that is possible to promote and increase the photodegradation rate of porphyrins and cyanine dyes in water through heterogeneous semiconductor photocatalysis. These two classes of dyes are sensitizers for photodynamic therapy of cancer and so after PDT cancer treatment these sensitizers and their photodegradation probes can reach water bodies. Zinctetraphenylphorpyrin did suffer significant photodegradation in the absence of a photocatalyst, but its presence definitely and efficiently promoted its substantial photodegradation. The studied cyanine dye experienced some photodegradation in the absence of photocatalyst and reached complete photodegradation in its presence during the time of the experiment. We can conclude that we successfully achieved the photodegradation of these two potential PDT agents in water and that the efficiency of the process is dependent of the irradiation time and on the amount of the photocatalyst used. This work demonstrates the possibility of using heterogeneous photocatalysis on the remediation of waste waters containing medicine residues from cancer PDT treatment.

\section{Acknowledgements}

The authors gratefully acknowledge the Fundação para a Ciência e Tecnologia (FCT), Portugal through projects PTDC/QUI/70123/2006, ERAMNT/0003/2009 and ERA-MNT/0004/2009. 


\section{References}

[1] Exall, K. A review of water reuse and recycling, with reference to Canadian practice and potential: 2. Applications. Water Research Journal of Canada, 39(1), pp. 13-28, 2004.

[2] Vigneswaran, S., Sundaravadiel, M. Recycle and Reuse of domestic wastewater. Wastewater recycle, reuse and reclamation, ed. Saravanamuthu, V., Eolss Publishers, Oxford, UK, 2004.

[3] Oliveira A. S., Saggioro E. M., Pavesi T., Moreira J. C., Vieira Ferreira L. F. Solar Photochemistry for Environmental Remediation - Advanced Oxidation Processes for Industrial Wastewater Treatment (Chapter 15), Molecular Photochemistry - Various Aspects, ed. Satyen Saha pp. 355380, 2012. ISBN: 978-953-51-0446-9.

[4] Güitekin, I., Ince, N.H., Synthetic endocrine disruptors in the environment and water remediation by advanced oxidation processes. Journal of Environmental Management, 85, pp. 816-832, 2007.

[5] Le-Minh, N., Khan, S.J., Drewes. J.E., Stuetz, R.M., Fate of antibiotics during municipal water recycling treatment processes. Water Research, $\mathbf{4 4}$, pp. 4295-4323, 2010.

[6] Fujishima A., Honda K., Electrochemical Photolysis of Water at a Semiconductor Electrode. Nature, 238, pp. 37, 1937.

[7] Oliveira A.S., Saggioro E.M., Barbosa N., Mazzei A., Vieira Ferreira L. F., Moreira J., Surface Photocatalysis: A Study of the Thickness of $\mathrm{TiO}_{2}$ Layers on the Photocatalytic Decomposition of Soluble Indigo Blue Dye. Rev. Chim., 62(4), pp. 462-468, 2011.

[8] Saggioro E., Oliveira A.S., Pavesi T., Maia C., Vieira Ferreira L. F., Moreira J., Use of Titanium Dioxide Photocatalysis on the Remediation of model textile wastewaters containing azo dyes. Molecules, 16, 1037010386, 2011.

[9] Socoteanu R., Boscencu R., Hirtopeanu A., Manda G., Oliveira A. S., Ilie M., Vieira Ferreira L. F., Trends in Interdisciplinary Studies Revealing Porphyrinic Compounds Multivalency Towards Biomedical Application, Biomedical Engineering - From Theory to Applications, ed. Reza FazelRezai, 2011. ISBN: 978-953-307-637-9.

[10] Boscencu R., Ilie M., Socoteanu R., Oliveira A.S., Constantin C., Neagu , M., Manda G., Vieira Ferreira L.F., Microwave Synthesis, Basic Spectral and Biological Evaluation of Some Copper (II) Mesoporphyrinic Complexes, Molecules, 15, pp. 3731-3743, 2010.

[11] Constantin, C, Neagu, M, Boscencu R., Hinescu, M. E., Oliveira, A. S.. Potential intracellular tracker capacity of novel synthetic metalloporphyrins, Toxicology Letters, 205, S61-S61, 2011.

[12] Vieira Ferreira L. F., Ferreira D. P., Oliveira A. S., Boscencu R., Socoteanu R., Ilie M., Constantin C., Neagu M., Synthesis, photophysical and cytotoxicity evaluation of A3B type mesoporphyrinic compounds. Dyes and Pigments, 95, pp. 296-303, 2012. 
[13] Santos P.F., Reis L.V., Almeida P., Oliveira A.S., Vieira Ferreira L.F., Efficiency of Singlet Oxygen Generation of Aminosquarylium Cyanines. J. Photochem. Photobiol., 163, pp. 267-269, 2004.

[14] Santos P.F., Reis L.V., Duarte I., Serrano J. P., Almeida P., Oliveira A.S., Vieira Ferreira L.F., Synthesis and photochemical evaluation of iodinated squarylium cyanine dyes. Helv. Chim. Acta, 88, pp. 1135, 2005.

[15] Vieira Ferreira L. F., Ferreira D. P., Duarte P., et al., Surface Photochemistry: 3,3'-Dialkylthia and Selenocarbocyanine Dyes Adsorbed onto Microcrystalline Cellulose, International Journal of Molecular Sciences, 13 (1), pp. 596-611, 2012. 